\title{
Understanding the Effect of Perceived Reasonableness on Customer Satisfaction in Relation to Moral Identity: An Abstract
}

\author{
Nobuyuki Fukawa
}

\begin{abstract}
A service provider standardizes various aspects of services to deliver those services ethically and satisfactorily. However, a service provider cannot plan for every contingency in designing its service specifications. Instead, a service provider could rely on morals of service personnel and customers and incorporate a certain level of flexibility within reason in its service design. The concept of "reasonableness" was proposed by Fukawa and Erevelles (2014) to explain such latitude that exists around expectations in the delivery of service. In this paper, we explore how customers' perceived reasonableness influences customer satisfaction in relation to moral identity of customers. First, we investigate whether the moral identity of customers influences perceived reasonableness. Second, we explore whether the effect of perceived reasonableness on customer satisfaction is explained by affective or cognitive processing. Our study indicates that moral identity is a critical determinant of perceived reasonableness. Furthermore, our study suggests that the perceived reasonableness influences customer satisfaction through affect rather than cognition.
\end{abstract}

References Available Upon Request

N. Fukawa $(\bowtie)$

Missouri University of Science and Technology, Rolla, MO, USA

e-mail: fukawan@mst.edu 